\title{
Intentional and unintentional medication non-adherence in hypertension: the role of health literacy, empowerment and medication beliefs
}

\author{
Lilla Náfrádi, Elisa Galimberti, Kent Nakamoto, Peter J. Schulz \\ Institute of Communication and Health, Università della Svizzera italiana, Lugano, Switzerland
}

\begin{abstract}
Significance for public health
Medication non-adherence is a critical public health issue, leading to increased health care costs, morbidity and mortality across several chronic conditions. Medication non-adherence is also one of the main obstacles to effective hypertension care, as between $20-50 \%$ of the patients do not follow appropriately their prescribed medication regimen. Despite the large body of empirical evidence available, relatively little is known about the potential differences between the determinants underpinning intentional versus unintentional nonadherence. Shedding light on the factors of medication non-adherence is inevitable to develop interventions and health campaigns addressing the issue. Thus, the present study quantitatively examines the patient-related factors of medication non-adherence to antihypertensive drugs, distinguishing between the determinants of deliberate versus non-deliberate non-adherence. Moreover, based on the outcomes, we advocate potential new approaches to address medication non-adherence in hypertension.
\end{abstract}

\section{Abstract}

Background: Medication non-adherence is a major public health issue, creating obstacles to effective treatment of hypertension. Examining the underlying factors of deliberate and non-deliberate non-adherence is crucial to address this problem. Thus, the goal of the present study is to assess the socio-demographic, clinical and psychological determinants of intentional and unintentional non-adherence.

Design and methods: A cross-sectional survey was conducted between March, 2015 and April, 2016. The sample consisted of hypertension patients holding at least one medical prescription $(\mathrm{N}=109)$. Measurements assessed patients' medication adherence, health literacy, empowerment, self-efficacy, medication beliefs, and patients' acceptance of their doctor's advice, socio-demographic and clinical characteristics.

Results: Patients who occasionally engaged in either intentional or unintentional non-adherence reported to have lower adherence selfefficacy, higher medication concern beliefs, lower meaningfulness scores and were less likely to accept the doctor's treatment recommendations. Patients who occasionally engaged in unintentional nonadherence were younger and had experienced more side effects compared to completely adherent patients. Adherence self-efficacy was a mediator of the effect of health literacy on patients' medication adherence and acceptance of the doctor's advice was a covariate.

Conclusions: Regarding the research implications, health literacy and adherence self-efficacy should be assessed simultaneously when investigating the factors of non-adherence. Regarding the practical implications, adherence could be increased if physicians i) doublecheck whether their patients accept the treatment advice given and ii) if they address patients' concerns about medications. These steps could be especially important for patients characterized with lower self-efficacy, as they are more likely to engage in occasional nonadherence.

\section{Introduction}

Medication non-adherence constitutes a significant obstacle to hypertension care with a prevalence between $20 \%$ and $50 \% .{ }^{1}$ Nonadherence is a complex phenomenon having a broad range of determinants, which can be grouped into patient-, physician-, medication- and health care system-related factors. ${ }^{2,3}$ In our study, we focus on the patient-related factors of medication non-adherence. In particular, we follow the precepts of the Health Empowerment Model, ${ }^{4}$ which advocates that health literacy and patient empowerment are interconnected but are also quite distinct predictors of various health behaviours. ${ }^{5}$ However, the interplay between health literacy and empowerment in a medication adherence context has not yet been tested.

Health literacy which refers to the degree to which individuals have the capacity to obtain, process, and understand basic health information and services needed to make appropriate health decisions ${ }^{6}$ is one of the patient-related factors of adherence. Two meta-analyses involving various patient groups unequivocally concluded that there was a statistically significant but weak relationship between health literacy and adherence. However, the evidence is mixed concerning cardiovascular patients, as some studies reported that low health literacy was associated with poor adherence, while others studies found no significant relationship. ${ }^{7,8}$ It is worth noting that the majority of the studies operationalized health literacy as basic numeracy and reading skills or health-related knowledge, but relatively little research has been done on its complex cognitive components, such as judgment skills. The term judgment skills refers to one's ability to examine one's own capabilities and limitations in order to make appropriate health decisions. ${ }^{9}$ The beneficial effect of proper judgment skills on disease management has been well documented, ${ }^{10,11}$ but its effect on medication adherence has not yet been addressed.

Psychological empowerment is conceived as the patients' willingness to act as autonomous actors taking increased responsibility for their own medical decision-making. ${ }^{12}$ Patient empowerment is a four dimensional concept: 1. Meaningfulness (refers to the value of activities worth investing energy in), 2. Impact (belief that one's actions can make a difference), 3. Self-determination (refers to making autonomous choices) and 4. Competence (belief in one's own capabilities to implement activities). ${ }^{4}$ Self-efficacy, which is closely related to the empowerment concept, is the most prominent determinant of adherence across conditions within the socio-cognitive and self-regulation theories. ${ }^{13}$ Medication beliefs are also important determinants of adherence. ${ }^{13-16}$ Those who endorse the belief that a certain medication is necessary for their health appear to be much more adherent than those who do not hold this belief. Conversely, people who endorse concern beliefs about their medication are less likely to be adherent compared to those who do not hold any concern beliefs. ${ }^{15}$

Beyond personal characteristics, certain aspects of the doctorpatient relationship can also influence patients' medication adherence. For instance, patients' acceptance of treatment advice, which is 
a proxy of the extent of patient's commitment, might also have an impact on medication adherence. Indeed, in an experimental setting, a positive link was seen between the participants' acceptance of medical advice and their intention to adhere to the given recommendation. ${ }^{17}$ However, whether this effect would hold true in a real-life setting has not yet been investigated.

The goal of the present article is to examine the clinical, cognitive and psychological determinants of medication adherence in hypertension. Moreover, we compared the determinants of intentional versus unintentional non-adherence, as the underpinning factors might significantly differ. ${ }^{18}$ We tested the assumption of the Health Empowerment Model, to see if health literacy and empowerment are distinct but intertwined predictors of medication adherence. Additionally, we examined the role of medication beliefs and self-efficacy in explaining adherence. In particular, we examined context-specific adherence self-efficacy beliefs, i.e. those closely linked to one's beliefs about one's own capacity to follow a prescribed medication regimen in challenging situations. ${ }^{19}$ Finally, we tested whether patients' acceptance of their doctor's medical advice can affect behavioural adherence.

\section{Design and Methods}

\section{Data collection}

The study applied a cross-sectional design. The questionnaires were pretested for face and content validity with a health care professional and patients respectively. The participants were recruited with the help of medical offices and hospitals. The data collection took place over the time period between March, 2015 and April, 2016. In order to participate in the study, patients had to fulfil the following criteria: having received hypertension treatment for a minimum of three months and being over 35 years of age. Before filling in the questionnaire, informed consent was obtained from the participants. We ensured anonymity to all respondents.

\section{Measures}

We set up measures of key constructs in our conceptual model as well as other potential factors of adherence.

The patients' behavioural non-adherence to drug regimens was measured on a 15-item scale developed based on the Medication Adherence Report Scale (MARS) ${ }^{20}$ We added ten items to the original 5 -item measure, ${ }^{21-23}$ which allowed us to create two separate sub-scales for intentional and unintentional non-adherence.

Respondents' functional health literacy was measured using the Newest Vital Sign (NVS). This is a performance-based measurement consisting of a nutritional product label accompanied by 6 questions. ${ }^{24}$
The Judgment Skills measure consists of four scenarios which describe typical everyday situations in disease management. ${ }^{10,11}$ The patients are instructed to indicate which behaviour they would most likely engage in when faced with the situation described in the scenario. As the potential behaviours differ in biomedical adequacy, the four options are then ranked. ${ }^{10,11}$

The Health Empowerment Scale was used based on the measure proposed by Spreitzer, ${ }^{25}$ adapted to the hypertension condition. The tool measures four facets of empowerment: Competence, Meaningfulness, Impact and Self-determination.

The Medication Adherence Self-Efficacy Scale (MASES) is a 13-item scale assessing patients' confidence in their ability to take their antihypertensive medication in a variety of situations. ${ }^{26}$

Beliefs about Medicines Questionnaire (BMQ) is an 11-item measure, consisting of two sub-scales. ${ }^{14,27}$ The Necessity subscale measures the perceived need for the medication(s), while the Concern sub-scale assesses concerns about potential negative effects such as dependence or side effects.

Participants' acceptance of treatment advice was measured with a modified 4-item scale assessing the extent to which the participants found the medical advice acceptable, clear and reasonable, and their level of satisfaction with the received information. ${ }^{17}$

We assessed the following socio-demographic variables: age, gender, educational attainment, health related education, employment status, marital status, family members living in the same household and linguistic background. Respondents were also asked to provide clinical information about the disease duration, and comorbidities. Regarding the medication regimen, the following information was obtained: the name of the prescribed medication(s), the frequency of its daily assumption and the side effects the respondents experienced.

\section{Analysis}

Descriptive statistics are reported as mathematical means and standard deviations. The Pearson correlation coefficient was used to determine the association between the scales. The outcomes of the adherent and non-adherent groups on the scales mentioned were compared with independent samples t-tests. Finally, a mediation analysis was performed.

\section{Results}

\section{The socio-demographic and clinical antecedents of medication adherence}

The sample consists of 109 hypertension patients (male: 69) holding at least one medical prescription. Table 1 shows the socio-demographic and clinical characteristics.

Table 1. The means and standard deviation of the socio-demographic and clinical characteristics of the sample (N=109)

\begin{tabular}{|c|c|c|c|c|c|c|c|c|}
\hline & \multicolumn{4}{|c|}{ Intentional (non-)adherence } & \multicolumn{4}{|c|}{ Unintentional (non-)adherence } \\
\hline & \multicolumn{2}{|c|}{$\begin{array}{l}\text { Complete } \\
\text { adherence } \\
(\mathrm{N}=56)\end{array}$} & \multicolumn{2}{|c|}{$\begin{array}{l}\text { Occasional } \\
\text { non-adherence } \\
(\mathrm{N}=53)\end{array}$} & \multicolumn{2}{|c|}{$\begin{array}{l}\text { Complete } \\
\text { adherence } \\
(\mathrm{N}=49)\end{array}$} & \multicolumn{2}{|c|}{$\begin{array}{l}\text { Occasional } \\
\text { non-adherence } \\
(\mathrm{N}=60)\end{array}$} \\
\hline & Mean & SD & Mean & SD & Mean & SD & Mean & SD \\
\hline Age (years) & 64.29 & 9.78 & 61.94 & 13.29 & 66.70 & 9.48 & 60.19 & 12.42 \\
\hline Illness duration (in years) & 11.97 & 9.33 & 10.91 & 9.27 & 11.85 & 8.55 & 11.15 & 9.95 \\
\hline Side effects $(0-10)$ & 1.61 & 1.53 & 2.06 & 1.93 & 1.19 & 0.9 & 2.34 & 2.07 \\
\hline Number of daily pills & 1.71 & 1.16 & 1.74 & 1.21 & 1.58 & 0.77 & 1.83 & 1.42 \\
\hline
\end{tabular}


The intentional and unintentional adherence scores were dichotomized due to the high level of adherence in the sample: participants were divided into two groups: complete adherence (respondents who achieved a maximum score on the scale, i.e. 100\% adherence) and occasional non-adherence (those who scored lower than the maximum). There was a moderate positive association between the intentional and unintentional adherence scales $(\mathrm{r}=0.493, \mathrm{P}<0.001)$.

Patients reporting occasional unintentional non-adherence behaviours are significantly younger than those who are completely adherent $(\mathrm{t}=-2.97 ; \mathrm{P}=0.004)$, while there was no age difference in the case of the intentionally adherent and non-adherent groups $(\mathrm{t}=-1.03 ; \mathrm{P}=0.29)$. Gender did not have a significant effect either on intentional or unintentional non-adherence.

People who were intentionally completely adherent versus intentionally occasionally non-adherent did not differ in terms of the duration of their illness, the side effects experienced, the number of medications being taken or the number of pill assumption per day. The results look extremely similar in the case of the unintentional non-adherence, with the exception that patients engaging in occasional unintentional nonadherence experienced significantly more side effects than adherent patients $(\mathrm{t}=-3.54 ; \mathrm{P}=0.001)$.

\section{The cognitive and psychological factors of medication adherence}

Patients who engaged occasionally in intentional non-adherence behaviour reported lower adherence self-efficacy $(\mathrm{t}=-4.54, \mathrm{P}<0.001)$, higher medication concern beliefs $(\mathrm{t}=2.13 ; \mathrm{P}=0.03)$, lower Empowerment Meaningfulness scores $(\mathrm{t}=-2.50 ; \mathrm{P}=0.01)$ and accepted to a lesser extent their doctor's treatment recommendations $(\mathrm{t}=2.29$; $\mathrm{P}=0.02$ ). No statistically significant differences have been found for the Empowerment Impact $(\mathrm{t}=0.01 ; \mathrm{P}=0.99)$, the Empowerment Selfdetermination $(\mathrm{t}=1.38 ; \mathrm{P}=0.16)$, the Empowerment Competence $(\mathrm{t}=$ $0.45 ; \mathrm{P}=0.65)$, the NVS $(\mathrm{t}=1.21 ; \mathrm{P}=0.22)$, the Judgment skills $(\mathrm{t}=-0.38$; $\mathrm{P}=0.40)$ or the $\mathrm{MBQ}$ Necessity scores $(\mathrm{t}=-1.05 ; \mathrm{P}=0.29)$.

The same pattern was found when analysing the unintentional nonadherence determinants: lower adherence self-efficacy $(\mathrm{t}=-3.15$, $\mathrm{P}=0.002)$, higher medication concern beliefs $(\mathrm{t}=-2.96 ; \mathrm{P}=0.004)$, lower Empowerment Meaningfulness scores $(\mathrm{t}=-2.32 ; \mathrm{P}=0.02)$ and lower acceptance of the doctor's treatment recommendations $(\mathrm{t}=-1.95$; $\mathrm{P}=0.05$ ) characterized those who engaged occasionally in unintentional non-adherence behaviours than the completely adherent patients. No statistically significant difference was found for the Empowerment
Impact $(\mathrm{t}=-1.75 ; \mathrm{P}=0.08)$, the Empowerment Self-determination $(\mathrm{t}=0.75 ; \mathrm{P}=0.45)$, the Empowerment Competence $(\mathrm{t}=0.60 ; \mathrm{P}=0.54)$, the NVS $(\mathrm{t}=-1.29 ; \mathrm{P}=0.19)$, the Judgment skills $(\mathrm{t}=-1.15 ; \mathrm{P}=0.25)$ or the MBQ Necessity scores $(\mathrm{t}=-1.11 ; \mathrm{P}=0.26)$ (Table 2$)$.

\section{Mediation analysis}

Based on the results of binomial logistic regression, a mediation analysis was performed to test the hypothesis that adherence self-efficacy (MASES) mediates the effect of functional health literacy (HL) on intentional non-adherence. Patients' acceptance of their doctor's advice was incorporated into the model as a covariate. No significant direct effect of HL was seen on intentional non-adherence $(b=-0.08$, $\mathrm{SE}=0.15, \mathrm{z}=-0.57, \mathrm{P}=0.56$ ). HL was a significant predictor of the MASES $(b=1.10, S E=0.36, t=3.02, P<0.003)$ and the MASES was a significant predictor of intentional non-adherence $(b=0.26, S E=0.07$, $\mathrm{t}=3.63, \mathrm{P}<0.001$ ). No significant effect of Patients' acceptance of the doctor's advice was found. The indirect effect was tested using a bootstrap estimation approach with 1000 samples, and analysis showed that the indirect coefficient was significant $(b=0.28, \quad S E=0.15$, $95 \% \mathrm{CI}=0.0963,0.7323)$. Finally, approximately $25 \%$ of the variance in the intentional non-adherence score was accounted for by the predictors (CoxSnell R2=0.2518; Nagelkrk R2=0.33). To sum up, these results support the mediation hypothesis that MASES can be considered to be a full mediator between HL and intentional non-adherence.

\section{Discussion and Conclusions}

Adherent patients were characterized as having a higher level of adherence self-efficacy, which is one of the most prominent predictors of adherence mentioned in literature. ${ }^{13}$ As for empowerment, the Meaningfulness dimension is the most important predictor, i.e. if patients consider their hypertension control activities worth investing energy in, they comply more with their medication regimens. Besides this, medication beliefs also play a role in influencing medication adherence, i.e. patients who endorse concern beliefs about their medication tend to adhere less to their medication regimens. Our findings are in accordance with previous meta-analysis which showed that in cases of cardiovascular disease, endorsing medication concern beliefs is a more important predictor of non-adherence than endorsing necessity beliefs. ${ }^{15}$ A qualitative review also reported that one of the main reasons behind hypertension patients' intentional non-adherence was

Table 2. The internal consistency and the means and standard deviations of the scales.

\begin{tabular}{|c|c|c|c|c|c|c|c|c|c|c|}
\hline \multirow[t]{3}{*}{ The scales } & \multirow{3}{*}{$\begin{array}{l}\text { Cronbach's } \\
\text { alpha }\end{array}$} & \multirow{3}{*}{$\begin{array}{l}\text { Score } \\
\text { range }\end{array}$} & \multicolumn{4}{|c|}{ Intentional (non-)adherence } & \multicolumn{4}{|c|}{ Unintentional (non-adherence) } \\
\hline & & & \multicolumn{2}{|c|}{$\begin{array}{l}\text { Complete } \\
\text { adherence }\end{array}$} & \multicolumn{2}{|c|}{$\begin{array}{l}\text { Occasional } \\
\text { non-adherence }\end{array}$} & \multicolumn{2}{|c|}{$\begin{array}{l}\text { Complete } \\
\text { adherence }\end{array}$} & \multicolumn{2}{|c|}{$\begin{array}{l}\text { Occasional } \\
\text { non-adherence }\end{array}$} \\
\hline & & & Mean & SD & Mean & SD & Mean & SD & Mean & SD \\
\hline NVS & 0.733 & $0-6$ & 4.42 & 1.62 & 4.03 & 1.74 & 4.46 & 1.54 & 4.05 & 1.78 \\
\hline MASES & 0.941 & $13-52$ & 48.84 & 3.65 & 43.64 & 7.68 & 48.39 & 4.66 & 44.61 & 7.25 \\
\hline MB Concern & 0.872 & $6-30$ & 14.11 & 5.46 & 16.58 & 5.6 & 13.36 & 4.68 & 16.78 & 5.91 \\
\hline MB Necessity & 0.842 & $5-25$ & 19.41 & 3.46 & 18.61 & 3.81 & 19.47 & 3.51 & 18.62 & 3.74 \\
\hline \multicolumn{11}{|l|}{ Empowerment } \\
\hline Meaningfulness & 0.612 & $1-7$ & 6.20 & 1.19 & 5.67 & 1.01 & 6.22 & 1.18 & 5.72 & 1.05 \\
\hline Impact & 0.902 & $1-7$ & 4.75 & 1.95 & 4.75 & 1.63 & 5.08 & 1.88 & 4.47 & 1.69 \\
\hline Self-determination & 0.821 & $1-7$ & 3.61 & 2.10 & 4.12 & 1.75 & 3.70 & 2.04 & 3.99 & 1.87 \\
\hline Competence & 0.629 & $1-7$ & 5.04 & 1.59 & 5.17 & 1.46 & 5.20 & 1.57 & 5.02 & 1.50 \\
\hline $\begin{array}{l}\text { Patients' acceptance } \\
\text { of the doctor's advice }\end{array}$ & 0.953 & $1-7$ & 6.56 & 0.75 & 6.09 & 1.21 & 6.56 & 0.78 & 6.16 & 1.15 \\
\hline Judgment skills & - & $1-4$ & 3.60 & 0.40 & 3.66 & 0.28 & 3.67 & 0.33 & 3.60 & 0.35 \\
\hline
\end{tabular}


a concern about drug side effects, e.g. developing an addiction. ${ }^{28}$ Additionally, adherent patients reported higher acceptance of their doctor's medical advice. Previously, a positive link was found between the participants' acceptance of medical advice and participants' intention to adhere to the given recommendation in an experiment. ${ }^{17}$ Our outcomes confirm that if the patients' believe that they have received clear and reasonable medical recommendations supported with arguments, this is then associated with the patients' self-reported behavioural medication adherence. The analysis did not reveal any significant difference in terms of the underpinning cognitive or emotional factors of intentional versus unintentional medication non-adherence. However, the findings have to be treated with caution, as intentional and unintentional adherence show a positive moderate correlation in our sample. We found significant differences in the socio-demographic and clinical factors, i.e. unintentionally occasionally non-adherent patients were younger and had experienced more side effects compared to the adherent patients, but there was no such difference in the case of intentional adherence. The finding that younger participants report higher unintentional non-adherence was unexpected and might be explained by lifestyle factors, as older patients might dedicate more time to following their medication regimen and there are many adherence aids available to and more likely to be used by the elderly, such as pill boxes and calendars. ${ }^{29}$ The results support the proposition that the clinical and socio-demographic determinants of deliberate and nondeliberate non-adherence should be investigated separately. ${ }^{18,30,31}$

Contrary to our expectations, health literacy was not a direct predictor of medication adherence. The mediation analysis revealed that health literacy is an indirect positive determinant of intentional medication adherence, and medication adherence self-efficacy mediates this relationship. Similarly, previous studies also reported that self-efficacy was a mediator of health literacy on adherence to self-care behaviours in kidney transplant patients ${ }^{32}$ and to medication adherence in HIV. ${ }^{33}$ These findings are in accordance with reviews which advocated that health literacy in itself cannot account for medication adherence, but that other variables should also be taken into consideration. ${ }^{7,8,34} \mathrm{We}$ suggest that self-efficacy is an important mediator of the effect of health literacy on medication adherence. Moreover, besides health literacy and self-efficacy, patients' acceptance of their doctor's advice was a covariate in the present model, so it is also an important factor in terms of medication adherence. To sum up, high level functional health literacy coupled with high adherence self-efficacy and a patient's satisfaction with their doctor's advice promote intentional medication adherence. However, judgment skills did not significantly influence the level of medication adherence. Thus, functional literacy skills seem to be more important in determining adherence, while judgment skills and other complex cognitive skills relative to health literacy concepts may tend to influence other aspects of disease management.

The present study is not without limitations. First of all, the major limitation of the study is that the prevalence of non-adherence is low in the sample. Based on the literature, patients are generally considered non-adherent if they take less than $80 \%$ of their medicines as ascertained by an objective adherence measure. ${ }^{3,35}$ Dichotomization is a common and a justified practice in research, ${ }^{36}$ patients are usually categorized as either adherent or non-adherent. However, in the present study the majority of the participants reported a high level of medication adherence; thus we applied another dichotomization: comparing the patients who reported complete adherence (having a maximum score on the adherence scale) and patients who reported occasional non-adherence (achieving less than the $100 \%$ score on the adherence measure). Taking into consideration this limitation, we argue that differentiating between completely adherent and occasionally non-adherent patients - providing a fine-grained assessment of differentiation between relatively high levels of adherence - should also be a relevant part of adherence research. Secondly, the principles for measuring intentional and unintentional adherence are not standardized yet; these trends showing a moderate positive association. Thirdly, our sample size was small. Forth, the study was cross-sectional, so we cannot derive conclusions about potential causal relationships. Finally, the patients engaging in occasional unintentional non-adherence experienced significantly more side effects compared to the adherent patients. The difference in the experienced side effects between patients might have had a stronger effect on their adherence level compared to the psychological and cognitive factors. Further qualitative research might explore how the differences in the clinical and psychosocial characteristics influenced patients' medication adherence.

This study highlights practical implications for promoting patients' medication adherence. First of all, it would be worth checking up on patients' acceptance of treatment advice after their medical consultation; and since a previous study showed that if patients have received reasonable arguments from their health care professionals supporting the treatment recommendations, this increases their acceptance of advice. ${ }^{17}$ Thus, motivating physicians and nurses to provide arguments can be a potential new approach to support shared decision making, ${ }^{17,37-39}$ and this in turn would increase patients' adherence. This is especially important for patients characterized with lower adherence self-efficacy, as these patients are more likely to engage in occasional non-adherence. Another potential way to increase medication adherence is through increasing patients' adherence self-efficacy. In particular, doctors and nurses might discuss certain situations with their patients which the patients appear to find particularly challenging when following their prescribed medication regimen. Talking over patients' medication concerns can also lessen their non-adherence behaviour. ${ }^{15,40}$ Regarding the research implications, health literacy and self-efficacy should be assessed simultaneously when investigating the factors of non-adherence. Moreover, patients' acceptance of their doctor's advice should also be taken into consideration. Besides this, future research with a greater sample size might further investigate the background factors and causes of intentional and unintentional non-adherence with separate samples only showing either intentional or unintentional non-adherence.

Correspondence: Lilla Náfrádi, Institute of Communication and Health, Università della Svizzera italiana, Via Giuseppe Buffi 13, 6904 Lugano, Switzerland.

Tel.: +41.58.666.4127 - Fax: +41.58 .666 .4647 .

E-mail: lilla.nafradi@usi.ch

Key words: medication adherence, hypertension, health literacy, self-efficacy, medication beliefs.

Funding: We would like to thank the Swiss National Science Foundation for their financial support (FNS 146 980) which made this study possible in the first place.

Acknowledgments: Special thanks go to the participants of the study. We gratefully acknowledge the support and collaboration of the physicians in recruiting the patients.

Contributions: NL, EG, KN,PJS study concept and design; NL, EG, PJS, statistical analysis; NL, EG, drafting of the manuscript; NL, EG, KN, PJS, critical revision of the manuscript for important intellectual content; KN, PJS, study supervision; NL,EG,KN, PJS, final approval.

Conflict of interest: the authors declare no potential conflict of interest.

Received for publication: 4 November 2016.

Accepted for publication: 5 December 2016.

○C Copyright L. Náfrádi et al., 2016

Licensee PAGEPress, Italy

Journal of Public Health Research 2016;5:762

doi:10.4081/jphr.2016.762 


\section{References}

1. Krousel-Wood M, Thomas S, Muntner P, Morisky D. Medication adherence: a key factor in achieving blood pressure control and good clinical outcomes in hypertensive patients. Curr Opin Cardiol 2004;19:357-62.

2. De Geest S, Sabaté E. Adherence to long-term therapies: evidence for action. Eur J Cardiovasc Nurs 2003;2:323.

3. Brown MT, Bussell JK. Medication adherence: WHO cares? Mayo Clin Proc 2011;86:304-14.

4. Schulz PJ, Nakamoto K. Health literacy and patient empowerment in health communication: The importance of separating conjoined twins. Patient Educ Couns 2013;90:4-11.

5. Camerini L, Schulz PJ, Nakamoto K. Differential effects of health knowledge and health empowerment over patients' self-management and health outcomes: a cross-sectional evaluation. Patient Educ Couns 2012;89:337-44.

6. Kindig DA, Panzer AM, Nielsen-Bohlman L. Health literacy: a prescription to end confusion. Washington, DC: National Academies Press; 2004.

7. Zhang NJ, Terry A, McHorney C. Impact of health literacy on medication adherence: a systematic review and meta-analysis. Ann Pharmacother 2014;48:741-51.

8. Miller TA. Health literacy and adherence to medical treatment in chronic and acute illness: a meta-analysis. Patient Educ Couns 2016;99:1079-86.

9. Rubinelli S, Schulz PJ, Nakamoto K. Health literacy beyond knowledge and behaviour: letting the patient be a patient. Int J Public Health 2009;54:307-31.

10. Dubowicz A, Schulz PJ. Functional validity of a judgment skills measure within the concept of health literacy for sleeping disorder patients. Int J Environ Res Public Health 2014;11:10868-82.

11. Londoño AMM, Schulz PJ. Influences of health literacy, judgment skills, and empowerment on asthma self-management practices. Patient Educ Couns 2015;98:908-17.

12. Schulz PJ, Nakamoto K. Bad literacy, the internet, and the limits of patient empowerment. Artif Intell 2011:65-69.

13. Holmes EF, Hughes D, Morrison VL. Predicting adherence to medications using health psychology theories: a systematic review of 20 years of empirical research. Value Heal 2014;17:863-76.

14. Horne R, Weinman J, Hankins M. The beliefs about medicines questionnaire: the development and evaluation of a new method for assessing the cognitive representation of medication. Psychol Heal 1999;14:1-24.

15. Foot H, La Caze A, Gujral G, Cottrell N. The necessity-concerns framework predicts adherence to medication in multiple illness conditions: a meta-analysis. Patient Educ Couns 2016;99:706-17.

16. Horne R, Chapman SCE, Parham R, et al. Understanding patients' adherence-related beliefs about medicines prescribed for longterm conditions: a meta-analytic review of the Necessity-Concerns Framework. PLoS One 2013;8:e80633.

17. Labrie NHM, Schulz PJ. The effects of general practitioners' use of argumentation to support their treatment advice: results of an experimental study using video-vignettes. Health Commun 2014;30:951-61.

18. Lehane E, McCarthy G. Intentional and unintentional medication non-adherence: A comprehensive framework for clinical research and practice? A discussion paper. Int J Nurs Stud 2007;44:1468-77.

19. Ogedegbe G, Mancuso CA, Allegrante JP, Charlson, M. E.Development and evaluation of a medication adherence self-effi- cacy scale in hypertensive African-American patients. J Clin Epidemiol 2003;56:520-9.

20. Horne R. The Medication Adherence Report Scale (MARS), Brighton, UK: University of Brighton, 2000.

21. Ediger JP, Walker JR, Graff L, et al. Predictors of medication adherence in inflammatory bowel disease. Am J Gastroenterol 2007;102:1417-26.

22. Ohm R, Aaronson LS. Symptom perception and adherence to asthma controller medications. J Nurs Scholarsh 2006;38:292-7.

23. Nicklas LB, Dunbar M, Wild M. Adherence to pharmacological treatment of non-malignant chronic pain: the role of illness perceptions and medication beliefs. Psychol Health 2010;25:601-15.

24. Weiss BD, Mays MZ, Martz W, et al. Quick assessment of literacy in primary care: The Newest Vital Sign. Ann Fam Med 2005;3:514-22.

25. Spreitzer GM. Psychological empowerment in the workplace: dimensions, measurement, and validation. Acad Manag J 1995;38:1442-65.

26. Fernandez S, Chaplin W, Schoenthaler AM, et al. Revision and validation of the medication adherence self-efficacy scale (MASES) in hypertensive African Americans. J Behav Med 2008;31:453-62.

27. Tibaldi G, Clatworthy J, Torchio E, et al. The utility of the Necessity-Concerns Framework in explaining treatment nonadherence in four chronic illness groups in Italy. Chronic Illn 2009;5:129-33.

28. Marshall IJ, Wolfe CD, McKevitt C. Lay perspectives on hypertension and drug adherence: systematic review of qualitative research. BMJ 2012;345:e3953.

29. Cramer JA. Enhancing patient compliance in the elderly. Drugs Aging 1998;12:7-15.

30. Atkins L, Fallowfield L. Intentional and non-intentional non-adherence to medication amongst breast cancer patients. Eur J Cancer 2006;42:2271-6.

31. Mo PKH, Mak WWS. Intentionality of medication non-adherence among individuals living with HIV/AIDS in Hong Kong. AIDS Care 2009;21:785-95.

32. Campbell ML. Effects of self-efficacy and heatlh literacy on adherence to self-care behaviors in kidney transplant patients. [Dissertation] University of Maryland, School of Pharmacy, Baltimore, Maryland, 2014.

33. Wolf MS, Davis TC, Osborn CY, et al. Literacy, self-efficacy, and HIV medication adherence. Patient Educ Couns 2007;65:253-60.

34. Ostini R, Kairuz T. Investigating the association between health literacy and non-adherence. Int J Clin Pharm 2014;36:36-44.

35. Nguyen TMU, Caze A L, Cottrell N. What are validated self-report adherence scales really measuring?: a systematic review. Br J Clin Pharmacol 2014;77:427-45.

36. DeCoster J, Iselin A-MR, Gallucci M. A conceptual and empirical examination of justifications for dichotomization. Psychol Methods 2009;14:349.

37. Salmon P. Argumentation and persuasion in patient-centred communication. Patient Educ Couns 2015;98:543-4.

38. Labrie NHM, Schulz PJ. Exploring the relationships between participatory decision-making, visit duration, and general practitioners' provision of argumentation to support their medical advice: results from a content analysis. Patient Educ Couns 2015;98:572-7.

39. Labrie N, Schulz PJ. Does argumentation matter? A systematic literature review on the role of argumentation in doctor-patient communication. Health Commun 2014;29:996-1008.

40. Easthall C, Song F, Bhattacharya D. A meta-analysis of cognitivebased behaviour change techniques as interventions to improve medication adherence. BMJ Open 2013;3:e002749. 